\title{
COLORECTAL CANCER
}

\section{Chemopreventive action of synthetic triterpenoids in CRC}

A new study has shown that synthetic triterpenoids, which are small molecules that inhibit expression of inflammatory mediators, are able to suppress colitis-associated colon cancer in mice. Translation of these findings to humans might have implications for chemoprevention in patients with IBD, who have a tenfold higher risk of colon cancer than age-matched control individuals.

By its very nature, colitis-associated colon cancer develops in an inflammatory environment, and is associated with downregulation of 15-hydroxyprostaglandin dehydrogenase (15-PGDH; an important tumour suppressor) and suppression of prostaglandin metabolism. "The development of novel agents that restore 15-PGDH expression represents a promising approach to chemoprevention of gastrointestinal tumorigenesis," explains John Letterio, corresponding author of the study.

The researchers had been intrigued by previous findings that inflammatory cytokines, such as TNF, could suppress the expression of 15-PGDH. These results led the team to question whether triterpenoids -with their anti-inflammatory and anticarcinogenic properties-could reverse this suppression. Thus, the researchers used mouse models of colitis-associated colon cancer to investigate the role of 15-PGDH expression and the effect of a specific synthetic triterpenoid (known as CDDO-Me) on this disease.

In a mouse model with T-cell-restricted deletion of Smad4, overexpression of proinflammatory cytokines was observed, leading to progressive loss of 15-PGDH and spontaneous development of colon cancer. Oral administration of CDDO-Me was reported to increase survival and suppress tumorigenesis. Notably, 15-PGDH expression was increased and production of proinflammatory cytokines was decreased in these mice. Similar suppression of tumorigenesis after administration of CDDO-Me was seen in a separate model of colon carcinogenesis (that is, in mice exposed to dextran sodium sulphate and azoxymethane).

Further experiments in human colon cells in vitro demonstrated that CDDO-Me was able to induce 15-PGDH expression; this induction was shown to be mediated by transforming growth factor $\beta$ (TGF- $\beta$ ) signalling, as it was blocked when cells were treated with TGF- $\beta$ receptor inhibitors. Induction of 15-PGDH by CDDO-Me was not observed in $S m a d 3^{-/-}$mice (Smad3 encodes a TGF- $\beta$ signalling intermediate).

"Our observations provide yet more compelling evidence that the application of triterpenoids for cancer chemoprevention should be pursued clinically," says Letterio. The study also highlights the importance of the TGF- $\beta$ pathway in the maintenance of intestinal homeostasis.

\section{Isobel Leake}

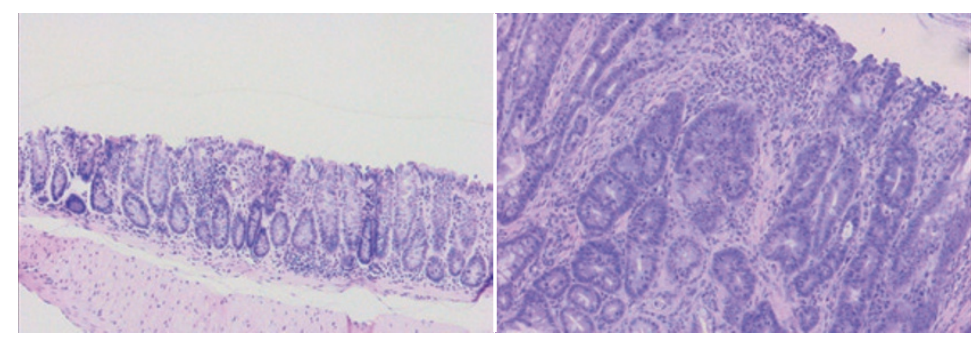

In mice with SMAD4-deficient T cells, intestinal inflammation is ameliorated in those treated with the synthetic triterpenoid CCDO-Me (left) compared with sesame oil (right). Image courtesy of J. Letterio.

Original article Choi, S. H. et al. Synthetic triterpenoid induces 15-PGDH expression and suppresses inflammation-driven colon carcinogenesis. J. Clin. Invest. doi:10.1172/JCI69672 